\title{
The effect of bezafibrate and omega-3 fatty acids on lymphocyte cytokine release and systemic inflammation in patients with isolated hypertriglyceridemia
}

\author{
Robert Krysiak • Anna Gdula-Dymek • \\ Boguslaw Okopien
}

Received: 2 November 2010 / Accepted: 10 May 2011 /Published online: 1 June 2011

(C) The Author(s) 2011. This article is published with open access at Springerlink.com

\begin{abstract}
Purpose The aim of this study was to compare the effects of fibrates and omega-3 fatty acids on lymphocyte secretory function and systemic inflammation in patients with isolated hypertriglyceridemia.

Methods The study included 107 patients with isolated hypertriglyceridemia who received bezafibrate $(200 \mathrm{mg}$ twice daily), omega-3 fatty acids (1 g twice daily) or placebo for 12 weeks. The lipid profile, fasting and 2-h postglucose load plasma glucose levels, homeostasis model assessment index (HOMA), plasma high-sensitivity Creactive protein (hsCRP) levels and lymphocyte release of interleukin-2, interferon- $\gamma$ and tumor necrosis factor- $\alpha$ were assessed at baseline, on the day of randomization, and after 4 and 12 weeks of treatment.

Results Both bezafibrate and omega-3 fatty acids reduced plasma triglyceride levels. Bezafibrate additionally decreased total and low-density lipoprotein-cholesterol levels and the HOMA and insignificantly decreased post-glucose load plasma glucose, as well as increased high-density lipoprotein-cholesterol. Bezafibrate treatment was associated with a reduction in lymphocyte release of interleukin-2, interferon- $\gamma$ and tumor necrosis factor- $\alpha$, which was accompanied by a reduction in plasma hsCRP levels. Omega-3 fatty acid did not significantly reduce lymphocyte cytokine release and plasma hsCRP. The anti-inflammatory effects of both
\end{abstract}

R. Krysiak $(\bowtie) \cdot$ A. Gdula-Dymek $\cdot$ B. Okopien

Department of Internal Medicine and Clinical Pharmacology,

Medical University of Silesia,

Medyków 18,

40-752 Katowice, Poland

e-mail: r.krysiak@interia.pl drugs did not correlate with their action on plasma lipids, but in the case of the former the effect was related to the improvement in insulin sensitivity.

Conclusion Our results indicate that bezafibrate is superior to omega-3 fatty acid in inhibiting systemic inflammation and lymphocyte secretory function.

Keywords Bezafibrate · Omega-3 fatty acids · Isolated hypertriglyceridemia $\cdot$ Lymphocytes $\cdot$ Proinflammatory cytokines $\cdot$ Low-grade inflammation

\section{Introduction}

Elevated triglyceride levels are modestly associated with the earlier development and accelerated progression of coronary artery disease [1]. In the Copenhagen Male Study [2], the Baltimore Observational Long Term Study [3] and the Bezafibrate Infarction Prevention study [4], which involved middle-aged and elderly men [2], subjects who underwent diagnostic coronary arteriography [3] and patients with coronary artery disease [4], respectively, the presence of hypertriglyceridemia was associated with an increased risk of cardiovascular morbidity and mortality. In a meta-analysis performed by Austin et al. [5], which included the results of 21 studies, each $90 \mathrm{mg} / \mathrm{dL}(1 \mathrm{mmol} / \mathrm{L})$ increase in plasma triglycerides was associated with a $32 \%$ increase in coronary artery disease events in men and a $76 \%$ increase in coronary artery disease events in women. However, adjustment for established coronary risk factors, especially for high-density lipoprotein (HDL) cholesterol, substantially attenuated the magnitude of the association between raised circulating triglyceride levels and increased coronary artery disease risk [6]. Moreover, because hyper- 
triglyceridemia is often associated with obesity, diabetes, metabolic syndrome, increased cholesterol levels, disturbed hemostasis and inflammation, some researchers assume that elevated triglyceride levels may be only a non-causal biomarker of future cardiovascular risk [7].

Activated $\mathrm{T}$ lymphocytes are present in all stages of human atherosclerotic lesion development, which implies their involvement in atherogenesis [8]. The major class of $\mathrm{T}$ cells found in the plaque, namely CD4+ helper cells, recognizes antigens associated with class II major histocompatibility complex (MHC) molecules, and mounts $\mathrm{T}$ helper cell type 1 responses with the secretion of proinflammatory cytokines, thus contributing to local inflammation and growth of the plaque [9]. Activated T lymphocytes stimulate macrophages of atherosclerotic lesions to produce metalloproteinases, which promote plaque instability and further implicate an immune response $[8,9]$. Therefore, inhibiting lymphocyte function may bring benefits to subjects with disturbed lipid metabolism, preventing or delaying the development of atherosclerosis and its complications.

To the best of our knowledge, no previous study has investigated the effect of any hypolipidemic agents on lymphocyte secretory function in this type of dyslipidemia. Although lymphocytes are involved in the pleiotropic effects of statins [10-12], these agents are not routinely used in subjects with isolated hypertriglyceridemia [7]. In turn, as our observations show, the strength of action of peroxisome proliferator-activated receptor-alpha (PPAR $\alpha)$ activators on lymphocyte cytokine release depends on the underlying disorder. The lymphocyte-suppressing effect of fibrates was evident in impaired glucose tolerance patients, absent in impaired fasting glucose individuals [14] and weak in mixed dyslipidemic subjects [10]. Therefore, the aim of the study reported here was to compare the effects of bezafibrate and omega- 3 fatty acids, two different treatment options for hypertriglyceridemia, on the lymphocyte secretory function in terms of their action on systemic inflammation in subjects with isolated elevation of plasma triglyceride levels. Interleukin (IL-2), interferon (IFN)- $\gamma$ and tumor necrosis factor-alpha (TNF- $\alpha$ ), all of which were assessed in our study, belong to the most important cytokines secreted by $\mathrm{T}$ cells and are regarded as relevant cardiovascular risk factors determining the risk of accelerated progression of atherosclerosis and the development of its complications $[13,15]$. To determine whether their action on lymphocyte cytokine release is associated with a global antiinflammatory effect, we measured high-sensitivity C-reactive protein (hsCRP) plasma levels, a highly sensitive marker of low-grade inflammation that has been shown in multiple prospective epidemiological studies to predict incident myocardial infarction, stroke, peripheral arterial disease, and sudden cardiac death $[16,17]$.

\section{Methods}

Subjects

Patients with recently diagnosed and previously untreated lipid metabolism abnormalities were eligible for inclusion in the study if they met the criteria of primary isolated hypertriglyceridemia [triglycerides between $200 \mathrm{mg} / \mathrm{dL}(2.3 \mathrm{mmol} /$ $\mathrm{L})$ and $500 \mathrm{mg} / \mathrm{dL}(5.7 \mathrm{mmol} / \mathrm{L})$; total cholesterol $<200 \mathrm{mg} / \mathrm{dL}$ $(5.2 \mathrm{mmol} / \mathrm{L})$; LDL-cholesterol $<130 \mathrm{mg} / \mathrm{dL}(3.4 \mathrm{mmol} / \mathrm{L})]$.

The exclusion criteria were: age $>70$ or $<35$ years; diabetes mellitus; any acute and chronic inflammatory processes; congestive heart failure; unstable coronary artery disease; myocardial infarction or stroke within 6 months preceding the study; moderate or severe arterial hypertension (grade 2 or 3 hypertension according to the European Society of Hypertension and European Society of Cardiology guidelines: systolic blood pressure $\geq 160 \mathrm{~mm} \mathrm{Hg}$ and/or diastolic blood pressure $\geq 100 \mathrm{~mm} \mathrm{Hg}$ ); impaired renal or hepatic function; concomitant treatment with other agents known either to affect plasma lipid levels or to interact with fibrates or omega-3 fatty acids; treatment with drugs that may affect inflammatory processes in the vascular wall (including glucocorticosteroids, nonsteroid anti-inflammatory drugs, calcium channel blockers, angiotensin converting enzyme inhibitors or angiotensin II receptor blockers) within 3 months preceding the study; ongoing hormonal replacement therapy or oral contraception; and poor patient compliance.

\section{Study design}

The study was approved by the Bioethical Committee of the Medical University of Silesia. Written informed consent was obtained from all study participants, and the study was performed according to the Declaration of Helsinki. All participants were informed about the benefits of a healthy diet and recommended to stop smoking, cease alcohol consumption and increase their physical activity to at least $30 \mathrm{~min}$ of moderate physical activity per day. Four weeks later, hypertriglyceridemic patients were randomized to bezafibrate (400 mg daily, $n=37$ ), omega-3 fatty acids ( $2 \mathrm{~g}$ daily, $n=37)$ or placebo $(n=33)$. Omega-3 fatty acids were administered in a form of a purified preparation consisting mainly of eicosapentaenoic acid (EPA) (465 mg) and docosahexaenoic acid (DHA) $(375 \mathrm{mg})$. Both drugs and placebo were administered twice daily in two equal doses. On the basis of the value of the homeostasis model assessment index (HOMA), each treatment group was divided into two subgroups comprising patients with normal or disturbed insulin sensitivity, respectively. Normal insulin sensitivity was arbitrary defined as a HOMA $<2.0$. If this value was exceeded, the patient was diagnosed as being insulin-resistant. 
The investigation of possible bezafibrate- or omega-3 fatty acid-induced adverse effects was performed every 2 weeks. Compliance was investigated at each visit by tablet counts and was considered to be satisfactory when the number of tablets taken by a patient ranged from 90 to $100 \%$.

The primary study objective was to evaluate the lymphocyte-suppressing and systemic anti-inflammatory effects of bezafibrate and omega- 3 fatty acids using a panel of inflammation markers: IL-2, IFN- $\gamma$, TNF- $\alpha$ and hsCRP.

\section{Laboratory assays}

The plasma levels of lipids, glucose and hsCRP and lymphocyte cytokine release were determined at baseline, on the day of randomization, and after 4 and 12 weeks of therapy. Venous blood samples were drawn from the antecubial vein after a 12-h overnight fast, in a quiet, temperature-controlled room $\left(24-25^{\circ} \mathrm{C}\right)$ between 0800 and 0900 hours (to avoid possible circadian fluctuations in the parameters studied). All blood samples were taken between 0800 and 0900 hours. All assays were carried out in duplicate to minimize analytical errors. The plasma lipid levels (total cholesterol, LDL-cholesterol, HDL-cholesterol and triglycerides) were determined colorimetrically using reagents purchased from bioMerieux (Marcy l'Etoile, France). LDL levels were measured directly. Plasma glucose concentrations were analyzed by a glucose oxidase method (Beckman, Palo Alto, CA). Plasma insulin was measured using a commercial radioimmunoassay kit (Linco Research, St Charles, MO). The HOMA was calculated from the following equation: fasting serum glucose $(\mathrm{mmol} / \mathrm{L}) \times$ fasting insulin level $(\mu \mathrm{U} / \mathrm{mL}) / 22.5$. The value of insulin used in this equation was the mean value of its concentrations in three different blood samples taken at 5-min intervals. Plasma levels of CRP were measured using a high-sensitivity monoclonal antibody assay (MP Biomedicals, Orangeburg, NY). The lower limit of sensitivity of this method was $0.1 \mathrm{mg} / \mathrm{L}$, while the intra-assay coefficients of variation were $4.3 \%$. T cells were cultured in triplicate as described earlier [10]. The release of IL-2, IFN- $\gamma$ and TNF- $\alpha$ by phytohemagglutinin-stimulated lymphocytes was measured using commercially available enzyme immunoassay methods (R\&D Systems, Minneapolis, MN). To avoid the freeze-thawing effect, each assay was performed on a single sample aliquot. The minimum detectable levels for IL-2, IFN- $\gamma$ and TNF- $\alpha$ were 8, 15 and $4.4 \mathrm{pg} / \mathrm{ml}$, respectively; the respective intra-assay coefficients of variation were $3.8,5.5$ and $4.6 \%$.

Power calculations

The sample size required for the study was calculated using Sample Power software (SPSS ver. 2.0; SPSS, Chicago, IL) based on data from our previous studies, assuming $80 \%$ power and a significance $(\alpha)$ level of 0.05 . According to the statistical power calculation, at least 90 patients would need to be randomized to detect a $20 \%$ difference in all components of the primary endpoint between pre- and posttreatment values within the same treatment group. Taking into account possible drop-outs and possible estimation and measurement inaccuracies, the number of participants (not counting the control group) was therefore increased to 107 patients.

\section{Statistical analysis}

The statistical analysis was performed using GraphPad Prism ver. 2.01 software (GraphPad Software, San Diego, CA) and Statistica 6.1 (StatSoft, Tulsa, OK). In all analyses, a $p$ value of $<0.05$ was considered to be statistically significant. The chi-squared test was used for all qualitative variables. Comparisons between the groups were performed using one-way analysis of variance (ANOVA) followed by Bonferroni's multiple comparison test (lipid profile, glucose), or with the Kruskall-Wallis test followed by the Mann-Whitney $U$ test (HOMA, hsCRP, cytokines). Student's paired $t$ test (lipid profile and plasma glucose) or the Wilcoxon test (HOMA, hsCRP and cytokines) were applied to compare pre-, inter- and posttreatment data within the same group. Correlations were studied using Kendall's tau test.

\section{Results}

Baseline characteristics

Main baseline characteristics of the included patients are summarized in Table 1. All treatment groups were well matched for general characteristics.

\section{Adverse effects}

In two patients treated with bezafibrate, therapy was discontinued prematurely because of headaches, nausea and abdominal pains. One subject receiving omega-3 fatty acids dropped out because of poor compliance with the study protocol. Two subjects randomized to omega-3 fatty acids discontinued medication because of nausea and vomiting. One patient who was randomized to placebo reported dizziness and declined to participate further in the study. No other serious adverse events occurred during the study. Baseline characteristics of the six subjects who left the study did not differ from those of the 101 patients who completed the study and were included in the final analyses. 
Table 1 Baseline characteristics of patients ${ }^{\mathrm{a}}$

\begin{tabular}{llll}
\hline Characteristics & \multicolumn{2}{l}{ Treatment groups } & \\
\cline { 2 - 4 } & Placebo & Omega-3 fatty acids & Bezafibrate \\
\hline Number of patients & 32 & 34 & 35 \\
Age (years) & $52.5 \pm 3.1$ & $53.1 \pm 3.5$ & $53.7 \pm 3.2$ \\
Men/women & $21 / 11$ & $22 / 12$ & $23 / 12$ \\
Body mass index (kg/m $\left.{ }^{2}\right)$ & $28.3 \pm 2.4$ & $28.6 \pm 2.8$ & $28.8 \pm 2.6$ \\
Smokers $(\%)$ & 31.3 & 35.3 & 31.4 \\
Mild hypertension (\%) & 25.0 & 26.5 & 28.6 \\
Stable coronary artery disease (\%) & 28.1 & 26.5 & 22.8 \\
Carotid artery atherosclerosis (\%) & 25.0 & 23.5 & 25.7 \\
Total cholesterol (mg/dl) & $168.9 \pm 7.3$ & $170.2 \pm 8.1$ & $169.8 \pm 8.0$ \\
LDL-cholesterol (mg/dl) & $101.3 \pm 4.1$ & $100.9 \pm 6.0$ & $102.3 \pm 3.7$ \\
HDL-cholesterol (mg/dl) & $37.7 \pm 1.2$ & $37.1 \pm 1.5$ & $37.3 \pm 1.4$ \\
Triglycerides (mg/dl) & $380.2 \pm 36.4$ & $378.2 \pm 39.2$ & $371.2 \pm 35.1$ \\
Fasting glucose (mg/dl) & $96.0 \pm 2.9$ & $95.9 \pm 2.6$ & $96.4 \pm 2.4$ \\
2-h post-glucose load plasma glucose (mg/dl) & $137.0 \pm 8.7$ & $136.9 \pm 5.8$ & $138.0 \pm 6.2$ \\
HOMA ratio & $3.7 \pm 0.2$ & $3.5 \pm 0.4$ & $3.8 \pm 0.5$ \\
hsCRP (mg/L) & $3.1 \pm 0.3$ & $3.3 \pm 0.5$ & $3.3 \pm 0.4$ \\
Interleukin-2 release (ng/mL) & $5.5 \pm 0.4$ & $5.9 \pm 0.8$ & $5.7 \pm 0.6$ \\
Interferon- $\gamma$ release (ng/mL) & $62.2 \pm 7.1$ & $65.2 \pm 8.0$ & $64.1 \pm 8.2$ \\
TNF- $\alpha$ release (pg/mL) & $400.1 \pm 42.5$ & $398.1 \pm 45.2$ & $402.3 \pm 38.5$ \\
\hline
\end{tabular}

LDL, Low-density lipoprotein; HDL, high-density lipoprotein; HOMA, homeostasis model assessment index; hsCRP, high-sensitivity C-reactive protein; TNF, tumor necrosis factor Where appropriate, data are given as the mean \pm standard deviation (SD)

${ }^{a}$ Only data of patients who completed the study were included in the final analyses lymphocyte cytokine release and plasma hsCRP between 4 and 12 weeks of treatment (Table 3).

There were no differences in the effect of omega-3 fatty acids on the HOMA, plasma hsCRP and lymphocyte cytokine release between "insulin-sensitive" and "insulin-resistant" patients (Table 4).

\section{Bezafibrate-treated group}

Bezafibrate treatment reduced plasma triglycerides by $35.8 \%(p<0.001)$ and $37.8(p<0.001)$, total cholesterol by $15.3(p<0.05)$ and $15.6 \%(p<0.05)$ and LDL-cholesterol by $13.1(p<0.05)$ and $14.3 \%(p<0.05)$, as well as increased HDL-cholesterol by $20.5(p<0.01)$ and $22.9 \%(p<0.01)$ after 4 and 12 weeks of treatment, respectively. The treatment also resulted in a reduction in the HOMA by $21.1 \%(p<0.05)$ after 4 weeks and by 50.0\% $(p<0.001)$ after 12 weeks of administration. Bezafibrate-induced changes in the HOMA were more evident at the end of the study than after 4 weeks of treatment. Bezafibrate treatment of isolated hypertriglyceridemic subjects did not change the fasting plasma glucose level, but it did reduce the 2-h post-challenge plasma glucose level, albeit nonsignficantly (after 4 weeks of treatment, by $8.8 \%, p=0.092$; at end of study: by $10.2 \%, p=0.074$ (Table 2).

After 4 weeks of bezafibrate administration to hypertriglyceridemic subjects, the drug reduced IL-2 release by $28.8 \%(p<0.01)$, IFN- $\gamma$ by $24.5 \%(p<0.05)$ and TNF- $\alpha$ by 
Table 2 The effect of bezafibrate and omega-3 fatty acids on the lipid profile and glucose homeostasis markers in hypertriglycericemic patients ${ }^{\mathrm{a}}$
Significance levels: ${ }^{*} p<0.05$, \#\# $p<0.01,{ }^{\# \#} p<0.001$ vs. respective value before randomization. ${ }^{*} p<0.05, * * p<0.01$, $* * * p<0.001$ vs. placebo-treated patients. $\wedge^{\wedge} p<0.05,{ }^{\wedge \wedge} p<0.001$ vs. omega-3 fatty acid-treated patients. ${ }^{\$ \$} p<0.01$ vs. respective value after 4 weeks of treatment

Data are given as the mean $\pm \mathrm{SD}$.

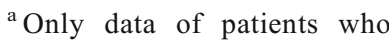
completed the study were included in the final analyses
Assay results

Treatment groups

Placebo Omega-3 fatty acids Bezafibrate

\begin{tabular}{|c|c|c|c|}
\hline \multicolumn{4}{|l|}{ Total cholesterol (mg/dL) } \\
\hline Baseline & $168.9 \pm 7.3$ & $170.2 \pm 8.1$ & $169.8 \pm 8.0$ \\
\hline Before randomization & $166.1 \pm 6.7$ & $169.2 \pm 4.9$ & $168.3 \pm 4.9$ \\
\hline After 4 weeks of treatment & $164.4 \pm 6.9$ & $173.6 \pm 6.8$ & $142.5 \pm 5.2^{\# * \wedge}$ \\
\hline After 12 weeks of treatment & $168.5 \pm 8.7$ & $174.4 \pm 6.8$ & $142.1 \pm 5.1^{\# * \wedge}$ \\
\hline \multicolumn{4}{|l|}{ LDL-cholesterol (mg/dL) } \\
\hline Baseline & $101.3 \pm 4.1$ & $100.9 \pm 6.0$ & $102.3 \pm 3.7$ \\
\hline Before randomization & $100.3 \pm 4.2$ & $99.5 \pm 5.7$ & $102.1 \pm 3.5$ \\
\hline After 4 weeks of treatment & $99.8 \pm 3.9$ & $102.3 \pm 4.6$ & $88.7 \pm 4.8^{\# * \wedge}$ \\
\hline After 12 weeks of treatment & $102.5 \pm 4.2$ & $104.2 \pm 5.0$ & $87.5 \pm 5.0^{\# * \wedge}$ \\
\hline \multicolumn{4}{|l|}{ HDL-cholesterol (mg/dL) } \\
\hline Baseline & $37.7 \pm 1.2$ & $37.1 \pm 1.5$ & $37.3 \pm 1.4$ \\
\hline Before randomization & $37.5 \pm 1.9$ & $38.2 \pm 1.4$ & $37.5 \pm 1.5$ \\
\hline After 4 weeks of treatment & $38.0 \pm 1.5$ & $39.3 \pm 1.7$ & $45.2 \pm 1.7^{\# \# \wedge}$ \\
\hline After 12 weeks of treatment & $37.8 \pm 1.4$ & $39.7 \pm 2.0$ & $46.1 \pm 1.6^{\# \# * \wedge}$ \\
\hline \multicolumn{4}{|l|}{ Triglycerides $(\mathrm{mg} / \mathrm{dL})$} \\
\hline Baseline & $380.2 \pm 36.4$ & $378.2 \pm 39.2$ & $371.2 \pm 35.1$ \\
\hline Before randomization & $366.2 \pm 30.3$ & $348.8 \pm 38.1$ & $351.2 \pm 31.8$ \\
\hline After 4 weeks of treatment & $346.5 \pm 43.2$ & $268.1 \pm 28.0^{\#}$ & $225.3 \pm 27.2^{\# \# \# * * *}$ \\
\hline After 12 weeks of treatment & $344.1 \pm 46.5$ & $240.1 \pm 29.7^{\# \# * *}$ & $218.2 \pm 28.3^{\# \# \# * * *}$ \\
\hline \multicolumn{4}{|l|}{ Fasting glucose $(\mathrm{mg} / \mathrm{dL})$} \\
\hline Baseline & $96.0 \pm 2.9$ & $95.9 \pm 2.6$ & $96.4 \pm 2.4$ \\
\hline Before randomization & $94.9 \pm 3.5$ & $94.6 \pm 3.1$ & $96.0 \pm 3.2$ \\
\hline After 4 weeks of treatment & $96.5 \pm 3.0$ & $94.0 \pm 2.9$ & $92.4 \pm 2.9$ \\
\hline After 12 weeks of treatment & $95.1 \pm 2.8$ & $95.1 \pm 3.7$ & $91.8 \pm 3.5$ \\
\hline \multicolumn{4}{|c|}{ 2-h post-glucose load plasma glucose (mg/dL) } \\
\hline Baseline & $137.0 \pm 8.7$ & $136.9 \pm 5.8$ & $138.0 \pm 6.2$ \\
\hline Before randomization & $138.2 \pm 8.0$ & $131.1 \pm 7.0$ & $137.2 \pm 5.3$ \\
\hline After 4 weeks of treatment & $138.8 \pm 8.3$ & $137.9 \pm 6.8$ & $125.1 \pm 4.9$ \\
\hline After 12 weeks of treatment & $136.5 \pm 7.5$ & $140.9 \pm 6.5$ & $123.2 \pm 5.5^{\wedge}$ \\
\hline \multicolumn{4}{|l|}{ HOMA } \\
\hline Baseline & $3.7 \pm 0.2$ & $3.5 \pm 0.4$ & $3.8 \pm 0.5$ \\
\hline Before randomization & $3.6 \pm 0.4$ & $3.4 \pm 0.3$ & $3.8 \pm 0.4$ \\
\hline After 4 weeks of treatment & $3.4 \pm 0.3$ & $3.7 \pm 0.4$ & $3.0 \pm 0.3^{\# \wedge}$ \\
\hline After 12 weeks of treatment & $3.5 \pm 0.3$ & $3.8 \pm 0.3$ & $1.9 \pm 0.2^{\# \# \# * * * \wedge \wedge \wedge \$ \$}$ \\
\hline
\end{tabular}

$23.6 \%(p<0.05)$ as well as decreased plasma hsCRP by $31.3 \%(p<0.001)$. At the end of the study, IL-2 release, IFN- $\gamma$ release, TNF- $\alpha$ release and plasma hsCRP, when compared to baseline, decreased by $52.5(p<0.001), 46.5$ $(p<0.001), 38.5(p<0.001)$ and 59.4\% $(p<0.001)$, respectively. Bezafibrate-induced changes in plasma hsCRP and lymphocyte cytokine release were more pronounced after 12 weeks than after 4 weeks of therapy (Table 3 ).

Bezafibrate affected the HOMA, plasma hsCRP and lymphocyte cytokine release more strongly in "insulinresistant" than "insulin-sensitive" patients (Table 4).

\section{Correlations}

At entry, there was a correlation between plasma hsCRP and lymphocyte secretory function $(r=0.48-0.58, p<$ $0.001)$, as well as between cytokine release or hsCRP and HOMA $(r=0.50-0.62, p<0.001)$. The effect of hypolipidemic agents on cytokine release and hsCRP did not correlate with their action on plasma triglycerides (bezafibrate-treated patients: $r=0.24$ between $\Delta \mathrm{IL}-2$ and $\Delta$ triglycerides, $r=0.32$ between $\Delta \mathrm{IFN}-\gamma$ and $\Delta$ triglycerides, $r=0.35$ between $\Delta \mathrm{TNF}$ $\alpha$ and $\Delta$ triglycerides, $r=0.26$ between $\Delta$ hsCRP and $\Delta$ trigly- 
Table 3 The effect on bezafibrate and omega- 3 fatty acids on plasma C-reactive protein and lymphocyte cytokine release in hypertriglyceridemic patients ${ }^{\mathrm{a}}$
Significance levels: ${ }^{*} p<0.05$, ${ }^{\# \#} p<0.01$, \#\#\# $p<0.001$ vs. respective value before randomization. ${ }^{*} p<0.05, * * p<0.01$, $* * * p<0.001$ vs. placebo-treated patients. ${ }^{\wedge \wedge} p<0.001$ vs. omega-3 fatty acid-treated patients. ${ }^{\$} p<0.05$,

$\$ \$<0.01, \$ \$ \$<0.001$ vs. respective value after 4 weeks of treatment

Data represent the mean \pm SD

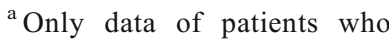
completed the study were included in the final analyses

\begin{tabular}{|c|c|c|c|}
\hline \multirow[t]{2}{*}{ Assay results } & \multicolumn{3}{|c|}{ Treatment groups } \\
\hline & Placebo & Omega-3 fatty acids & Bezafibrate \\
\hline \multicolumn{4}{|l|}{ hsCRP (mg/L) } \\
\hline Baseline & $3.1 \pm 0.3$ & $3.3 \pm 0.5$ & $3.3 \pm 0.4$ \\
\hline Before randomization & $3.0 \pm 0.4$ & $3.1 \pm 0.4$ & $3.2 \pm 0.3$ \\
\hline After 4 weeks of treatment & $3.2 \pm 0.5$ & $2.6 \pm 0.4$ & $2.2 \pm 0.2^{\# \# \# * * *}$ \\
\hline After 12 weeks of treatment & $2.9 \pm 0.5$ & $2.5 \pm 0.4$ & $1.3 \pm 0.2^{\# \# \# * * * \wedge \wedge \wedge \$ \$ \$}$ \\
\hline \multicolumn{4}{|l|}{ Interleukin-2 release $(\mathrm{ng} / \mathrm{mL})$} \\
\hline Baseline & $5.5 \pm 0.4$ & $5.9 \pm 0.8$ & $5.7 \pm 0.6$ \\
\hline Before randomization & $5.6 \pm 0.6$ & $5.5 \pm 1.1$ & $5.8 \pm 0.8$ \\
\hline After 4 weeks of treatment & $5.7 \pm 0.8$ & $4.6 \pm 0.5$ & $4.2 \pm 0.6^{\# \# * *}$ \\
\hline After 12 weeks of treatment & $5.8 \pm 0.8$ & $4.5 \pm 0.8$ & 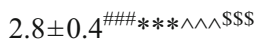 \\
\hline \multicolumn{4}{|l|}{ Interferon- $\gamma$ release $(\mathrm{ng} / \mathrm{mL})$} \\
\hline Baseline & $62.2 \pm 7.1$ & $65.2 \pm 8.0$ & $64.1 \pm 8.2$ \\
\hline Before randomization & $65.0 \pm 8.0$ & $64.7 \pm 8.5$ & $63.9 \pm 7.1$ \\
\hline After 4 weeks of treatment & $65.2 \pm 7.5$ & $53.2 \pm 6.2$ & $48.2 \pm 7.8^{\# * *}$ \\
\hline After 12 weeks of treatment & $64.8 \pm 9.7$ & $52.4 \pm 5.9$ & $34.2 \pm 7.9^{\# \# \# * * * \wedge \wedge \wedge \$}$ \\
\hline \multicolumn{4}{|l|}{$\mathrm{TNF}-\alpha$ release $(\mathrm{pg} / \mathrm{mL})$} \\
\hline Baseline & $400.1 \pm 42.5$ & $398.1 \pm 45.2$ & $402.3 \pm 38.5$ \\
\hline Before randomization & $395.7 \pm 38.1$ & $395.2 \pm 37.2$ & $395.3 \pm 36.4$ \\
\hline After 4 weeks of treatment & $394.2 \pm 40.1$ & $320.1 \pm 31.5$ & $302.2 \pm 28.5^{\#} *$ \\
\hline After 12 weeks of treatment & $404.3 \pm 38.4$ & $318.6 \pm 29.2$ & $243.1 \pm 22.5^{\# \# \# * * * \wedge \wedge}$ \\
\hline
\end{tabular}

cerides - all non-significant; omega-3 fatty acid-treated patients: $r=0.31$ between $\Delta \mathrm{IL}-2$ and $\Delta$ triglycerides, $r=0.28$ between $\Delta \mathrm{IFN}-\gamma$ and $\Delta$ triglycerides, $r=0.34$ between $\Delta \mathrm{TNF}$ $\alpha$ and $\Delta$ triglycerides, $r=0.29$ between $\Delta$ hsCRP and $\Delta$ triglycerides - all non-significant) nor with the action of these agents on the remaining lipid/lipoprotein fractions (bezafibratetreated patients: $r=0.04-0.22$, all non-significant; omega-3 fatty acid-treated patients: $r=0.06-0.24$, all non-significant). In subjects treated with bezafibrate, the effect of this drug on cytokine release correlated with its action on hsCRP $(r=0.47-$ $0.61, p<0.001)$. Moreover, in this group of patients, bezafibrate-induced changes in hsCRP and cytokine release correlated with the changes in the HOMA $(r=0.51-0.58, p<$ $0.001)$.

\section{Discussion}

Our study is the first to show that bezafibrate is able to inhibit lymphocyte release of proinflammatory cytokines, while the effect of omega-3 fatty acids appears to be much less pronounced. The anti-inflammatory actions of the former drug observed in our study, which explain the reduction in whole blood TNF- $\alpha$ and IL- 6 production in bezafibrate-treated hypertiglyceridemic subjects [18, 19], are in line with results from large clinical trials. Bezafibrate was found to reduce the incidence of coronary events in young male survivors of myocardial infarction participating in the Bezafibrate Coronary Artherosclerosis Intervention Trial (BECAIT) [20] and to reduce the combined incidence of probable ischemic changes on resting electrocardiogram and documented myocardial infarction in individuals with type 2 diabetes free of clinical cardiovascular disease who were enrolled in the St. Mary's, Ealing, Northwick Park Diabetes Cardiovascular Disease Prevention (SENDCAP) study [21]. Moreover, our results may partially explain why in the Bezafibrate Intervention Prevention study, the most evident clinical benefits (a reduction in the primary endpoint by $39.5 \%$ ) were observed in patients with elevated $(>200 \mathrm{mg} / \mathrm{dL})$ plasma triglyceride levels [4, 22]. Interestingly, the clinical benefits of bezafibrate have been better documented than those of fenofibrate [23, 24], although the latter drug is more commonly used in clinical practice [7]. It is worth mentioning that in our previous study [10], fenofibrate only insignificantly reduced lymphocyte cytokine release in subjects with increased circulating levels of both triglycerides and cholesterol. This clinical superiority of bezafibrate may be attributed to its additional action on other $(\beta / \delta$ and $\gamma)$ types of PPARs $[25,26]$.

As our results show, a fibrate-induced reduction in plasma hsCRP, also observed previously by other authors $[18,27,28]$, partially results from a decrease in lymphocyte cytokine release. A lymphocyte-suppressing effect may be also responsible for a fibrate-induced decrease in the 
Table 4 Effect of bezafibrate and omega-3 fatty acids on the HOMA, plasma hsCRP and lymphocyte cytokine release in insulin-sensitive and insulin-resistant patients with isolated hypertriglyceridemia ${ }^{a}$

\begin{tabular}{|c|c|c|c|c|}
\hline \multirow[t]{2}{*}{ Assay results } & \multicolumn{2}{|c|}{ Omega-3 fatty acid-treated patients } & \multicolumn{2}{|c|}{ Bezafibrate-treated patients } \\
\hline & $\begin{array}{l}\text { Insulin-sensitive } \\
\text { subjects }(n=15)\end{array}$ & $\begin{array}{l}\text { Insulin-resistant } \\
\text { subjects }(n=19)\end{array}$ & $\begin{array}{l}\text { Insulin-sensitive } \\
\text { subjects }(n=17)\end{array}$ & $\begin{array}{l}\text { Insulin-resistant } \\
\text { subjects }(n=18)\end{array}$ \\
\hline \multicolumn{5}{|l|}{ HOMA } \\
\hline Baseline & $1.4 \pm 0.3$ & $5.2 \pm 0.6 * * *$ & $1.5 \pm 0.2$ & $5.9 \pm 0.5^{* * *}$ \\
\hline Before randomization & $1.3 \pm 0.2$ & $5.1 \pm 0.5^{* * *}$ & $1.6 \pm 0.2$ & $5.8 \pm 0.4 * * *$ \\
\hline After 4 weeks of treatment & $1.3 \pm 0.2(-0.2)$ & $5.6 \pm 0.6(+9.8)^{* * *}$ & $1.4 \pm 0.3$ & $4.5 \pm 0.3(-22.4)^{* * * \#}$ \\
\hline $\begin{array}{l}\text { After } 12 \text { weeks of treatment } \\
\text { hsCRP }(\mathrm{mg} / \mathrm{L})\end{array}$ & $1.4 \pm 0.2(-7.7)$ & $5.2 \pm 0.4(+2.0)^{* * *}$ & $1.3 \pm 0.2$ & 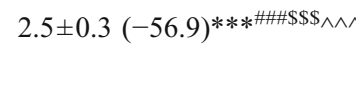 \\
\hline Baseline & $2.9 \pm 0.3$ & $3.6 \pm 0.5$ & $2.9 \pm 0.3$ & $3.7 \pm 0.6$ \\
\hline Before randomization & $2.8 \pm 0.4$ & $3.3 \pm 0.4$ & $2.6 \pm 0.4$ & $3.8 \pm 0.5$ \\
\hline After 4 weeks of treatment & $2.3 \pm 0.3(-17.8)$ & $2.8 \pm 0.3(-22.2)$ & $2.1 \pm 0.2(-19.2)$ & $2.3 \pm 0.3(-39.5)^{\# \# \#}$ \\
\hline After 12 weeks of treatment & $2.2 \pm 0.3(-21.4)$ & $2.8 \pm 0.4(-22.2)$ & $1.6 \pm 0.2(-38.4)^{\# \#}$ & $1.0 \pm 0.3(-73.6)^{* \# \# \$ \$ \$ \wedge \wedge \wedge}$ \\
\hline \multicolumn{5}{|l|}{ Interleukin-2 release (ng/mL) } \\
\hline Baseline & $4.6 \pm 0.5$ & $6.9 \pm 0.8 * * *$ & $4.5 \pm 0.7$ & $6.8 \pm 0.4 * * *$ \\
\hline Before randomization & $4.3 \pm 0.5$ & $6.5 \pm 0.7 * * *$ & $4.4 \pm 0.5$ & $7.0 \pm 0.7 * * *$ \\
\hline After 4 weeks of treatment & $3.8 \pm 0.5(-11.6)$ & $5.3 \pm 0.6(-18.5)^{* * *}$ & $3.4 \pm 0.5(-22.7)^{\#}$ & $5.0 \pm 0.7(-28.6)^{* * * \# \#}$ \\
\hline After 12 weeks of treatment & $3.7 \pm 0.4(-14.0)$ & $5.2 \pm 0.5(-19.8)^{* * *}$ & $3.1 \pm 0.6(-29.5)^{\# \#}$ & $2.5 \pm 0.4(-64.3)^{\# \# \# \$ \$ \wedge \wedge \wedge}$ \\
\hline \multicolumn{5}{|l|}{ Interferon- $\gamma$ release $(\mathrm{ng} / \mathrm{mL})$} \\
\hline Baseline & $54.3 \pm 7.8$ & $73.8 \pm 6.4 * * *$ & $53.1 \pm 6.2$ & $74.5 \pm 7.4 * * *$ \\
\hline Before randomization & $53.2 \pm 7.5$ & $73.7 \pm 8.3^{* * *}$ & $52.8 \pm 7.3$ & $74.3 \pm 6.9 * * *$ \\
\hline After 4 weeks of treatment & $43.1 \pm 5.8(-19.0)$ & $61.2 \pm 6.5(-17.0)^{* * *}$ & $41.3 \pm 5.7(-21.8)^{\#}$ & $54.7 \pm 5.0(-26.4)^{* * \# \#}$ \\
\hline After 12 weeks of treatment & $42.9 \pm 6.5(-19.4)$ & $59.9 \pm 6.8(-18.7)^{* * *}$ & $38.4 \pm 8.0(-27.3)^{\#}$ & $30.1 \pm 5.3(-59.5)^{\# \# \# \$ \$ \$ \wedge \wedge \wedge}$ \\
\hline \multicolumn{5}{|l|}{$\mathrm{TNF}-\alpha$ release $(\mathrm{pg} / \mathrm{mL})$} \\
\hline Baseline & $338.5 \pm 34.4$ & $445.1 \pm 40.4 * *$ & $345.9 \pm 30.2$ & $458.6 \pm 40.2 * * *$ \\
\hline Before randomization & $337.4 \pm 32.1$ & $440.8 \pm 35.6^{* *}$ & $340.2 \pm 31.1$ & $455.6 \pm 41.7^{* * *}$ \\
\hline After 4 weeks of treatment & $274.3 \pm 25.1(-18.7)$ & $356.2 \pm 50.2(-19.2)^{* *}$ & $274.5 \pm 29.1(-19.3)$ & $328.4 \pm 35.5(-27.3)^{\# \#}$ \\
\hline After 12 weeks of treatment & $273.9 \pm 30.5(-18.8)$ & $353.8 \pm 40.7(-19.7)^{* *}$ & $259.8 \pm 25.3(-23.6)^{\#}$ & $225.9 \pm 41.2(-50.4)^{\# \# \# \$ \wedge \wedge \wedge}$ \\
\hline
\end{tabular}

${ }^{*} p<0.05,{ }^{* *} p<0.01, * * * p<0.001$ vs. insulin-sensitive patients in the same treatment group; ${ }^{\#} p<0.05,{ }^{\# \#} p<0.01$, ${ }^{\# \#} p<0.001$ vs. respective value before randomization; ${ }^{\$} p<0.01,{ }^{\$ \$} p<0.001$ indicate that the effect was stronger than after the 4-week treatment; $p<0.001$ indicates the effect of bezafibrate at the end of the study was stronger than the effect of omega-3 fatty acids in the same subgroup of patients

Each value represents the mean $\pm \mathrm{SD}$. Values in parenthesis represent the percentage change from the respective values on the day of randomization ${ }^{a}$ Only data of patients who completed the study were included in the final analyses

plasma content of TNF- $\alpha$ and IL-6, which was accompanied by a decrease in plasma fibrinogen, in hypertriglyceridemic patients with concomitant mild or moderate hypertension and in severe cardiovascular disease-free hypertriglyceridemic patients receiving fibrate therapy [18, 27]. These findings provide evidence that inhibition of the lymphocyte secretory function contributes to the systemic anti-inflammatory actions of bezafibrate. Interestingly, in our unpublished observations, the extent of a bezafibrateinduced reduction in hsCRP in hypertriglyceridemic patients correlated with the effect of this agent on monocyte cytokine release (Krysiak et al., submitted), indicating that the global anti-inflammatory impact of bezafibrate is secondary to its action on various types of cells present in atherosclerotic lessons. Our results have shown that the lymphocyte-suppressing and systemic anti-inflammatory actions of bezafibrate increased with time, and this fact may explain a time-dependent reduction in the incidence of the primary endpoint in bezafibrate-treated coronary artery disease patients [22].

In addition to reducing lymphocyte cytokine release, bezafibrate exhibited a beneficial, although weak effect on glucose metabolism, increasing insulin sensitivity and slightly reducing post-glucose load plasma glucose. This finding is in agreement with our previous observations, which revealed that fenofibrate improved glucose metabolism markers in type 2 diabetic patients [29]; more recently, it has also been shown to improve glucose metabolism markers in 
subjects with prediabetes [30]. Because the effect on the HOMA and post-challenge plasma glucose was exhibited by two drugs that differ from each other in their physicochemical and pharmacokinetic properties $[25,26]$, this effect likely represents a "class effect" of fibrates and may explain why bezafibrate treatment reduced the incidence of diabetes by $30 \%$ in subjects with a baseline glucose level between 110 and $125 \mathrm{mg} / \mathrm{dl}$ [22].

In comparison with bezafibrate, the effect of omega- 3 fatty acids was much more limited, and in the case of cytokine release and plasma hsCRP, the effect did not reach the level of statistical significance. This finding is in agreement with the results of a meta-analysis of randomized controlled trials, which showed only small changes in plasma hsCRP in patients treated with omega-3 fatty acids [31]. Taking into consideration the role of both lymphocytes and proinflammatory cytokines in the process of atherogenesis $[8,9,13$, 15], a weak lymphocyte-suppressing effect of omega-3 fatty acids may be, however, clinically relevant. It may have contributed to a reduction in the number of coronary artery disease events found in the Japan EPA Lipid Intervention Study (JELIS) [32] and to a reduction in the number of deaths from any cause, nonfatal myocardial infarction and nonfatal stroke observed in the Gruppo Italiano per lo Studio della Sopravvivenza nell'Infarto (GISSI)-Prevenzione trial [33]. However, it may be too weak to prevent the incidence of sudden cardiac death, fatal and nonfatal myocardial infarction, coronary artery bypass grafting and percutaneous coronary interventions [32]. Unlike bezafibrate, the effect of omega-3 fatty acids on lymphocyte cytokine release and systemic inflammation was almost maximal after only 4 weeks of treatment, which may in part explain why in the GISSI-Prevenzione trial benefits of omega-3 fatty acid therapy appeared shortly after its initiation [33]. Because omega-3 fatty acids did not affect glucose homeostasis markers while the anti-inflammatory effect did not differ between "insulin-sensitive" and "insulin-resistant" subjects, it seems that their administration brings similar clinical benefits to patients with and without glucose metabolism abnormalities.

The lack of correlation between the extent of the reduction in lipid levels and the effect of hypolipemic treatment on $\mathrm{T}$ lymphocyte function indicates that the observed changes in inflammatory mediators cannot be explained by triglyceridelowering or other lipid- modulating features of the two agents investigated and that the latter represent pleiotropic effects. Lymphocyte-suppressing and global anti-inflammatory effects of bezafibrate correlated with the degree of reduction in the HOMA. Moreover, these effects were stronger in "insulinresistant" than in "insulin-sensitive" subjects. This finding is in agreement with the results of the Veterans Affairs High-Density Lipoprotein Intervention Trial (VA-HIT), which revealed that gemfibrozil reduced cardiovascular events and cardiovascular mortality mainly in type 2 diabetic subjects or in nondiabetic patients with elevated plasma insulin [34]. One of the factors linking the restoration of insulin sensitivity and lymphocytesuppressing and CRP-lowering effects of bezafibrate are free fatty acids, which are the endogenous ligands for PPAR [35]. We make this assumption because in our previous observations [36], fenofibrate action on monocyte secretory function and plasma hsCRP correlated with a fenofibrate-induced reduction in the circulating levels of free fatty acids. In turn, omega-3 fatty acids may produce their weak lymphocytesuppressive and systemic anti-inflammatory effects indirectly by displacing omega- 6 fatty acids, including arachidonic acid, in the cell membrane and reducing the production of other proinflammatory factors, including prostaglandins, thromboxanes and leukotrienes [37].

This study is not free of a number of limitations. Firstly, we recruited a relatively small number of participants. It is possible that the effect of omega- 3 fatty acids would reach the level of statistical significance if more patients were included. Secondly, patients were treated with moderate doses of either bezafibrate or omega-3 fatty acids. Therefore, we cannot exclude that these agents, particularly omega-3 fatty acids, produce even stronger lymphocyte-suppressing and systemic anti-inflammatory effects if they are administered at higher doses or in combination. Finally, as we assessed global secretory function of lymphocytes, there may be some differences between various subsets of $\mathrm{T}$ cells with respect to their response to bezafibrate and omega- 3 fatty acids.

To summarize, this study has shown that bezafibrate produces both lipid-improving and anti-inflammatory effects in hypertriglyceridemic subjects, while the analog effects of low dose omega-3 fatty acids are much more moderate. Our findings indicate that bezafibrate is superior to omega-3 fatty acids in inhibiting systemic inflammation and lymphocyte secretory function.

Conflict of interest disclosure The authors state that they have no conflict of interest.

Declaration of funding This study was supported by the State Committee for Scientific Research (grant number 2 P05F 036 29).

Open Access This article is distributed under the terms of the Creative Commons Attribution Noncommercial License which permits any noncommercial use, distribution, and reproduction in any medium, provided the original author(s) and source are credited.

\section{References}

1. Brunzell JD (2007) Hypertriglyceridemia. N Engl J Med 357:1009-1017 
2. Jeppesen J, Hein HO, Suadicani P, Gyntelberg F (1998) Triglyceride concentration and ischemic heart disease: an eight-year follow-up in the Copenhagen Male Study. Circulation 97:1029-1036

3. Miller M, Seidler A, Moalemi A, Pearson TA (1998) Normal triglyceride levels and coronary artery disease events: the Baltimore Coronary Observational Long-Term Study. J Am Coll Cardiol 31:1252-1257

4. BIP (Bezafibrate Infarction Prevention) Study Group (2000) Secondary prevention by raising HDL cholesterol and reducing triglycerides in patients with coronary artery disease: the Bezafibrate Infarction Prevention (BIP) study. Circulation 102:21-27

5. Austin MA, Hokanson JE, Edwards KL (1998) Hypertriglyceridemia as a cardiovascular risk factor. Am J Cardiol 81(Suppl 4A):7B-12B

6. Sarwar N, Danesh J, Eiriksdottir G et al (2007) Triglycerides and the risk of coronary heart disease: 10158 incident cases among 262525 participants in 29 Western prospective studies. Circulation 115:450-458

7. Yuan G, Al.-Shali KZ, Hegele RA (2007) Hypertriglyceridemia: its etiology, effects and treatment. Can Med Assoc J 176:1113-1120

8. Weyand CM, Younge BR, Goronzy JJ (2008) T cells in arteritis and atherosclerosis. Curr Opin Lipidol 19:469-477

9. Hansson GK (2009) Inflammatory mechanisms in atherosclerosis. J Thromb Haemost 7(Suppl 1):328-331

10. Okopień B, Krysiak R, Kowalski J et al (2004) The effect of statins and fibrates on interferon-gamma and interleukin-2 release in patients with primary type II dyslipidemia. Atherosclerosis 176:327-335

11. Yilmaz A, Reiss C, Weng A et al (2006) Differential effects of statins on relevant functions of human monocyte-derived dendritic cells. J Leukoc Biol 79:529-538

12. Leung BP, Sattar N, Crilly A et al (2003) A novel antiinflammatory role for simvastatin in inflammatory arthritis. J Immunol 170:1524-1530

13. Tedgui A, Mallat Z (2006) Cytokines in atherosclerosis: pathogenic and regulatory pathways. Physiol Rev 86:515-581

14. Krysiak R, Okopien B (2010) The effect of fenofibrate on lymphocyte cytokine release in patients with impaired fasting glucose and impaired glucose tolerance: a preliminary report. Atherosclerosis 213:325-328

15. Schroecksnadel K, Frick B, Winkler C, Fuchs D (2006) Crucial role of interferon-gamma and stimulated macrophages in cardiovascular disease. Curr Vasc Pharmacol 4:205-213

16. Ridker PM (2007) Inflammatory biomarkers and risks of myocardial infarction, stroke, diabetes, and total mortality: implications for longevity. Nutr Rev 65(12 Pt 2):S253-S259

17. Kinlay S, Egido J (2006) Inflammatory biomarkers in stable atherosclerosis. Am J Cardiol 98:2P-8P

18. Jonkers IJ, Mohrschladt MF, Westendorp RG, van der Laarse A, Smelt AH (2002) Severe hypertriglyceridemia with insulin resistance is associated with systemic inflammation: reversal with bezafibrate therapy in a randomized controlled trial. Am J Med 112:275-280

19. Mohrschladt MF, Weverling-Rijnsburger AW, de Man FH et al (2000) Hyperlipoproteinemia affects cytokine production in whole blood samples ex vivo. The influence of lipid-lowering therapy. Atherosclerosis 148:413-419

20. de Faire U, Ericsson CG, Nilsson J, Grip L, Svane B, Hamsten A (1997) Retardation of coronary atherosclerosis: the Bezafibrate Coronary Atherosclerosis Intervention Trial (BECAIT) and other angiographic trials. Cardiovasc Drugs Ther 11(Suppl 1):257-263

21. Elkeles RS, Diamond JR, Poulter C et al (1998) Cardiovascular outcomes in type 2 diabetes. A double-blind placebo-controlled study of bezafibrate: the St. Mary's, Ealing, Northwick Park Diabetes Cardiovascular Disease Prevention (SENDCAP) Study. Diabetes Care 21:641-648

22. Goldenberg I, Benderly M, Goldbourt U (2008) Update on the use of fibrates: focus on bezafibrate. Vasc Health Risk Manag 4:131141

23. Keech A, Simer RJ, Barter P et al (2005) Effects of long-term fenofibrate therapy on cardiovascular events in 9795 people with type 2 diabetes mellitus (the FIELD study): randomised controlled trial. Lancet 366:1849-1861

24. ACCORD Study Group, Ginsberg HN, Elam MB, Lovato LC et al (2010) Effects of combination lipid therapy in type 2 diabetes mellitus. N Engl J Med 362:1563-1574

25. Backes JM, Gibson CA, Ruisinger JF, Moriarty PM (2007) Fibrates: what have we learned in the past 40 years? Pharmacotherapy 27:412424

26. Gross B, Staels B (2007) PPAR agonists: multimodal drugs for the treatment of type-2 diabetes. Best Pract Res Clin Endocrinol Metab 21:687-710

27. Ye P, Li JJ, Su G, Zhang C (2005) Effects of fenofibrate on inflammatory cytokines and blood pressure in patients with hypertriglyceridemia. Clin Chim Acta 35:229-232

28. Ikewaki K, Tohyama J, Nakata Y, Wakikawa T, Kido T, Mochizuki S (2004) Fenofibrate effectively reduces remnants, and small dense LDL, and increases HDL particle number in hypertriglyceridemic men - a nuclear magnetic resonance study. J Atheroscler Thromb 11:278-285

29. Pruski M, Krysiak R, Okopien B (2009) Pleiotropic action of short-term metformin and fenofibrate treatment, combined with lifestyle intervention, in type 2 diabetic patients with mixed dyslipidemia. Diabetes Care 32:1421-1424

30. Krysiak R, Gdula-Dymek A, Bachowski R, Okopien B (2010) Pleiotropic effects of atorvastatin and fenofibrate in metabolic syndrome and different types of prediabetes. Diabetes Care 33:2266-2270

31. Balk EM, Lichtenstein AH, Chung M, Kupelnick B, Chew P (2006) Lau J (2006) Effects of omega-3 fatty acids on serum markers of cardiovascular disease risk: a systematic review. Atherosclerosis 189:19-30

32. Yokoyama M, Origasa H, Matsuzaki M, Japan EPA lipid intervention study (JELIS) Investigators et al (2007) Effects of eicosapentaenoic acid on major coronary events in hypercholesterolaemic patients (JELIS): a randomised open-label, blinded endpoint analysis. Lancet 369:1090-1098

33. Gruppo Italiano per lo Studio della Sopravvivenza Nell'Infarto Miocardico (1999) Dietary supplementation with -3 polyunsaturated fatty acids and vitamin $\mathrm{E}$ after myocardial infarction: results of the GISSI-Prevenzione trial. Lancet 354:447-455

34. Rubins HB, Robins SJ, Collins D et al (2002) Diabetes, plasma insulin, and cardiovascular disease: subgroup analysis from the Department of Veterans Affairs high-density lipoprotein intervention trial (VA-HIT). Arch Intern Med 162:2597-2604

35. Keating GM, Ormrod D (2002) Micronised fenofibrate: an updated review of its clinical efficacy in the management of dyslipidaemia. Drugs 62:1909-1944

36. Okopien B, Krysiak R, Herman ZS (2006) Effects of short-term fenofibrate treatment on circulating markers of inflammation and hemostasis in patients with impaired glucose tolerance. J Clin Endocrinol Metab 91:1770-1778

37. Simopoulos AP (2008) The importance of the omega-6/omega-3 fatty acid ratio in cardiovascular disease and other chronic diseases. Exp Biol Med 233:674-688 